\title{
Anamorphic Signature Identification Based on Correlative Specific Area of Restored and Segmented Image with Transformable Gridding
}

\author{
Ning Wang ${ }^{1,2}$ Guoqiang Han ${ }^{1}$ \\ ${ }^{1}$ School of Computer Science and Engineering, South China University of Technology, Guangzhou 510641, P. R. \\ China \\ ${ }^{2}$ Guangzhou Public Security Bureau, Guangzhou 510030, P. R. China
}

\begin{abstract}
This paper proposes a method to identify the anamorphic signature. The signature is set on a transformable gridding. Moving the gridding in opposite direction with anamorphic direction, the anamorphic signature is restored. Meanwhile, the restored signature is segmented into equal-area sections by the gridding. The specific area is the ratio of strokes area to section area for each section. It is constant for every signature despite for the anamorphic signature. The correlation coefficient between identified and standard signature is calculated according to their specific areas. The high correlation coefficient expresses the high similarity between the identified and standard signature.
\end{abstract}

Keywords: Anamorphic signature, Identification, Specific area, Correlation coefficient, Transformable gridding

\section{Introduction}

Signature identification is a hot topic for many researchers so far. But it is also difficult because the usable characters are few and the characteristic of writer is often intentionally or undesignedly concealed. In natural signature identification, time-consuming Markov algorithm is often used [1]. The method of SVM and HMM are also used to verify the offline signature [2]-[3]. These methods are unfit for anamorphic signature because it stresses the common process and ignores many features of signature image yet. The effective feature is the key of successful signature identification [4]-[5].

In this paper, the anamorphic signature is classified as four classes-longitudinal narrowing or broadening, latitudinal narrowing or broadening, upincline or down-incline, and incline-narrowing or incline-broadening. First, these anamorphic signatures are set on special transformable gridding platform. By moving the gridding, they can be corrected into natural and normal shape and style to increase the successful probability of signature identification. Then, the restored signature is segmented into some equal-area quadrate sections by the gridding. In natural style of signature, the ratio of the strokes area of every section to the area of section is relative constant for every signature. This ratio is called as the specific area. The specific area is a constant in spite of the natural signature becomes anamorphic. The method of specific area is a new pattern of signature identification. Moreover, the local characteristic of specific area of sections makes up of the whole structural characteristic of signature. If the specific area is used to study the correlation of identified signature and standard signature, it will be a matching template of structure of signature image. It is similar with DNA replication template of biology cell. Such pattern recognition can be called as DNA template replication mechanism. By calculation of correlation coefficient of specific area between identified signature and standard signature, the anamorphic signature identification can be completed. This method can evaluate the similarity of signatures very well. The high similarity of signature images expressed the high matching based on template mechanism [6]-[7].

\section{Anamorphic signature image processing}

\subsection{Signature image acquisition and binalization}

85 natural and anamorphic Signatures are collected as the studied objects. Then, the professional scanner with not less than 1200dpi optical resolving power is prepared to acquire signature image. The scan parameter is same for all signature images. By 
experiment, 1000ppi resolving power, mode of reflection and default parameter are optimal condition. All signature images are saved as bitmap format.

The captured signature image is binarized by improved form-analysis of histogram to get binary image [8]. First, signature is transformed into gray image by scanner. The gray image of signature is binalized with global threshold method because signature image is relative simple. The optimal threshold is got by improved form-analysis of histogram automatically [9]-[10]. Let $f(x, y)$ be the original image, $g(x, y)$ be the binary image, $t$ be the optimal threshold. So we have the following formula to binalize the signature image.

$$
g(x, y)= \begin{cases}1 & f(x, y) \geq t \\ 0 & f(x, y)<t\end{cases}
$$

\subsection{Anamorphic signature restoration and segmentation with gridding}

\subsubsection{The type of anamorphic signature}

According to the analysis about all kinds of signatures, anamorphic signature mainly includes the following four classes. The first is longitudinal narrowing or broadening signature. The second is latitudinal narrowing or broadening signature. The third is upincline or down-incline signature. The fourth is inclinenarrowing or incline-broadening signature (see the following Figures).

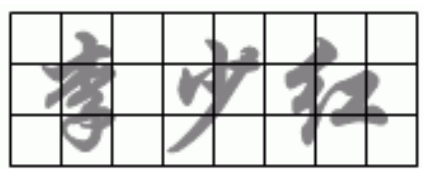

Fig. 1: Natural and segmented signature with gridding.

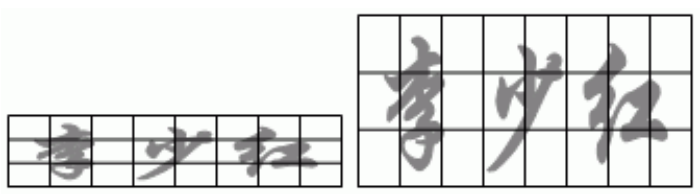

Fig. 2: Longitudinal narrowing or broadening and segmented signature with gridding.
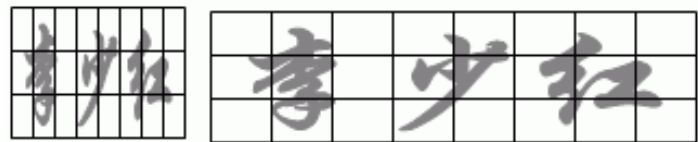

Fig. 3: Latitudinal narrowing or broadening and segmented signature with gridding.

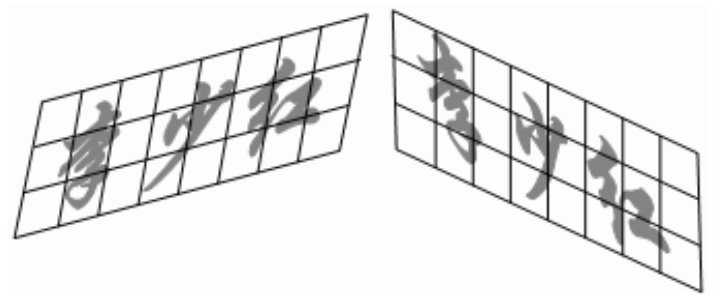

Fig. 4: Up-incline or down-incline and segmented signature with gridding.

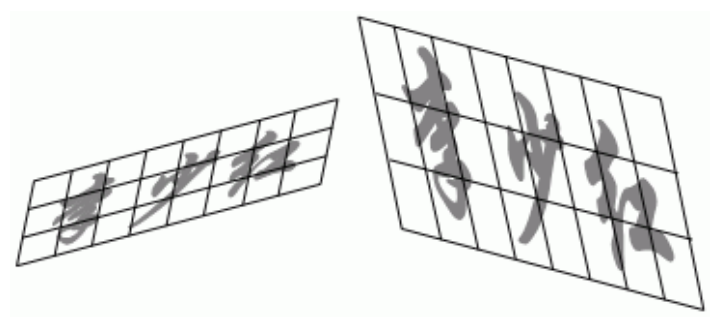

Fig. 5: Incline-narrowing or incline-broadening and segmented signature with gridding.

\subsubsection{Anamorphic signature restoration}

To simplify and normalize the identification of anamorphic signature, all anamorphic signatures need to be restored to natural and normal signature. These anamorphic signature images are set on a transformable gridding. By the moving, rotation, compressing or extending of transformable gridding in opposite direction with anamorphic direction, these anamorphic signatures are restored to natural style basically. The restored signature keeps quadrate shape to be segmented easily (see Fig. 1).

\subsubsection{Restored signature segmentation with equal- area-section gridding}

For feature extraction, the restored signature is segmented into some equal-area quadrate sections by the quadrate gridding. Analogously with matrix, the sequence of sections of signature image is from left to right and from top to bottom (see Fig. 1). For example, to three-character Chinese signature, every character is segmented into 9 equal-area sections on $3 \times 3$ mode if there is definite interval between characters. Otherwise, as a whole, the signature is segmented into 27 equalarea sections on $3 \times 9$ mode.

\subsubsection{The characteristic of signature classification and segmentation}

Every signature has some characteristic knows from others from the whole to the local of signature. They show the signature is exclusive and are mainly evidence of signature identification. These characteristics can be studied solely. According to the importance of characteristic, they are classified into 
four classes-highly remarkable characteristic, remarkable characteristic, appreciably remarkable characteristic, common characteristic. They are also segmented into some equal-area section by suitable quadrate gridding respectively.

\section{Signature feature extraction}

\subsection{The specific area of standard signature}

The specific area of standard signature is the ratio of the area of strokes of every section to the area of equalarea quadrate section. Let $\sigma_{0}$ be the area of rectangular gridding, $\sigma_{i j}$ be the area of section, $s_{i j}$ be the area of strokes of every section, $\lambda_{i j}$ be the specific area of every section. We can get $\lambda_{i j}$ by formula (2) and (3).

$$
\begin{aligned}
\sigma_{i j} & =\sigma_{0} / n \\
\lambda_{i j} & =s_{i j} / \sigma_{i j}(i=1,2, \cdots, m \quad j=1,2, \cdots, n)
\end{aligned}
$$

Where $i$ is the sequence and number of the type of section, $j$ is the sequence and number of section.

The specific area of characteristic area also can be calculated according to above formulas.

\subsection{The specific area of identified signature}

The calculation of specific area of identified signature is same with standard signature. We use the variables with superscript "\#" to express the eigenvalues of identified signature. Let ${ }^{\#} \sigma_{0}$ be the area of rectangular gridding, ${ }^{\#} \sigma_{i j}$ be the area of section, ${ }^{\#} s_{i j}$ be the area of strokes of section, ${ }^{\#} \lambda_{i j}$ be the specific area of every section. Thus, we have the following formulas.

$$
\begin{aligned}
& { }^{*} \sigma_{i j}={ }^{\#} \sigma_{0} / n \\
& { }^{*} \lambda_{i j}={ }^{\#} s_{i j} /{ }^{\#} \sigma_{i j} \quad(i=1,2, \cdots, m \quad j=1,2, \cdots, n)
\end{aligned}
$$

\section{The method of signature identification}

\subsection{The correlation coefficient calculation}

The theory of correlation can be used to evaluate the degree of similarity of digital images. In this paper, the method of correlation coefficient is used to finish the anamorphic signature identification. According to the method of calculation of correlation coefficient, we use the specific area to calculate the correlation coefficient between standard signature and identified signature (see formula (6)) [11].

$$
* r=\frac{\sum(x-\bar{x})(y-\bar{y})}{\sqrt{\sum(x-\bar{x})^{2}} \sqrt{\sum(y-\bar{y})^{2}}}
$$

To simplify the calculation, let $r$ be the absolute value of $* r$. Thus, we have the following condition.

$$
r=\left|{ }^{*} r\right|
$$

The correlation coefficient of specific area of characteristic area is also calculated by formula (6)

\subsection{The weight of correlation coefficient}

According to the correlation coefficient $r_{i}$ of whole signature and local characteristic, we use $A_{i}$ to represent all features of signature identification. Let $A_{1}$ be the whole signature, $A_{2}$ be the highly remarkable characteristic, $A_{3}$ be the remarkable characteristic, $A_{4}$ be the appreciably remarkable characteristic, $A_{5}$ be the common characteristic. The weight of $A_{i}$ can be got in terms of their importance in signature identification (see Table 1).

$\begin{array}{cccccc} & A_{1} & A_{2} & A_{3} & A_{4} & A_{5} \\ \text { Weight } & 0.50 & 0.20 & 0.15 & 0.10 & 0.05\end{array}$

Table 1: The weight of correlation coefficient $r_{i}$.

\subsection{The standard of signature identification}

Let $v$ be the value of evaluation. It is calculated by following formula.

$$
v=\sum A_{i} r_{i} \quad(i=1,2,3,4,5)
$$

According to Table 1, the concrete $v$ is got by formula (9).

$$
v=0.5 r_{1}+0.2 r_{2}+0.15 r_{3}+0.1 r_{4}+0.05 r_{5}
$$

With the statistic method and knowledge, the standard of signature correlation identification can be worked out. If the correlation coefficient is between 0.95 and 0.99 , we think the identified signature be clearly similar with the standard signature. If the correlation coefficient is more than 0.99 , we think the identified signature is real. If the correlation coefficient is between 0.68 and 0.95 , we think the identified signature be questionably similar with the standard signature. It will be difficult to do the signature 
identification. If the correlation coefficient is less than

following Table 2 shows it.

0.68 , we think the identified signature is bogus. The

\begin{tabular}{|ccccc|}
\hline The concept of identification & dissimilarity & questionable similarity & obvious similarity & absolute similarity \\
\hline The standard value $v$ & $v<0.68$ & $0.68 \leq v \leq 0.95$ & $0.95<v \leq 0.99$ & $v>0.99$ \\
\hline
\end{tabular}

Table 2: The standard of signature identification based on the correlation coefficient.

\section{Anamorphic signature identification experiment}

According to above method, the experiment of 46 someone's latest two-time signatures and 39 imitated signatures is completed very well. The result of experiment is ideal (see Table 3 ).

\begin{tabular}{|cccccc|}
\hline The source of signature & The number of signatures & $v<0.68$ & $0.68 \leq v \leq 0.95$ & $0.95<v \leq 0.99$ & $v>0.99$ \\
\hline Someone's signature & $46(100 \%)$ & 0 & 0 & $2(4.3 \%)$ & $44(95.7 \%)$ \\
Imitated signature & $39(100 \%)$ & $32(82.1 \%)$ & $3(7.7 \%)$ & $4(10.2 \%)$ & 0 \\
\hline
\end{tabular}

Table 3: The result of experiment of anamorphic signature identification.

The result of experiment shows that this method is feasible and effective.

\section{Framework of anamorphic signature identification}

Fig. 6 shows the framework of program of anamorphic signature identification.

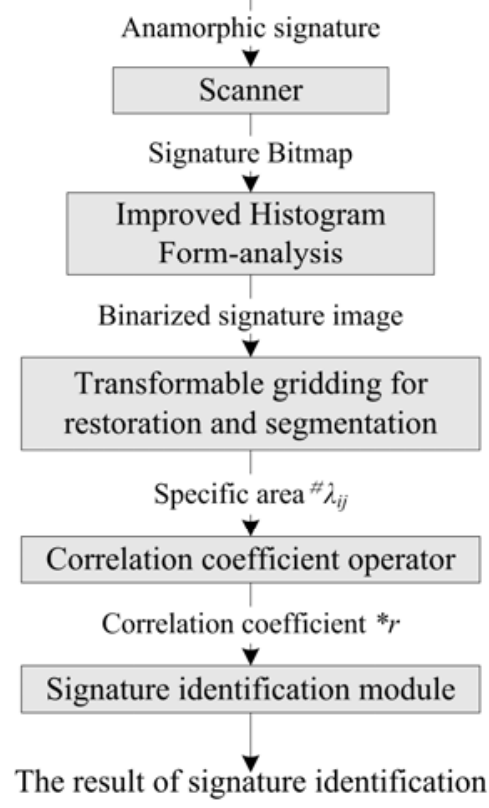

Fig. 6: The framework of anamorphic signature identification .

The program adopts the component mode to realize anamorphic signature identification based on the .NET of Microsoft. It is very advantageous to increase the capability of every module and improve the stability and security of program.

\section{Discussion}

This paper proposed a new method of anamorphic signature identification. By restoration and feature extraction, anamorphic signature can be identified satisfyingly. According to the shape and distribution of signature, anamorphic signature is classified into four types-longitudinal narrowing or broadening, latitudinal narrowing or broadening, up-incline or down-incline, and incline-narrowing or inclinebroadening. These anamorphic signatures are set on a transformable gridding. They can be restored to natural and normal shape to extract feature easily through moving, rotation, compressing or extending of gridding in opposite direction with anamorphic direction. On this condition, anamorphic signatures are comparable just.

Experiment shows, after the restored anamorphic signature is segmented into some equal-area sections by quadrate gridding, the area of strokes of every section can be proportionately changed with the area of section in anamorphic signature. The specific area just is the ratio of the area of strokes of every section to the area of section. So the specific area is relatively constant. Moreover, it is the structural eigenvalue of signature. Therefore, the linear correlation of identified signature and standard signature becomes a pattern of signature identification. The degree of correlation is the standard of matching degree based on template. The high correlation expresses the result of high matching. This matching mode is similar with DNA replication template of biology cell. Such DNA 
template replication mechanism can be used widely in signature identification and other images recognition.

\section{Conclusions}

Signature identification based on similar specific area of restored and segmented image with transformable gridding is an ideal method of anamorphic signature identification. The restoration of signature and the calculation of correlation coefficient of specific area is the key.

The specific area of whole signature and local characteristic is relative constant. It is very important eigenvalue to identify the anamorphic signature successfully. It can also become a mode of other image identification.

\section{Acknowledgement}

This work is partially supported by National Nature Science Foundation of China (Grant No. 60573019), the Natural Science Foundation of Guangdong (Grant No. 05103541), and the Scientific Research Foundation of Guangzhou Public Security Bureau.

\section{References}

[1] R.S. Kashi, J. Hu, W.L. Nelson and W. Turin, On-line Handwritten Signature Verification Using Hidden Markov Model Features, Document Analysis and Recognition, Proceedings of the Fourth International Conference on, pp.253-257, 1997.

[2] H. R. Lu, W.Y. Wang, C. Wang and Q. Zhuo, Off-line Chinese Signature Verification Based on Support Vector Machines, Pattern Recognition Letter, 26:2390-2399, 2005.

[3] E.J. R. Justino, F. Bortolozzi and R. Sabourin, The Interpersonal and Intrapersonal Variability Influences on Off-line Signature Verification Using HMM, Proceedings of Computer Graphics and Image Processing, pp.197-202, 2002.

[4] M.E. Munich and P. Perona, Visual identification by signature tracking, IEEE Transactions on Pattern Analysis and Machine Intelligence, 25:200-217,2003.

[5] Y.Y. Tang, L.T. Tu and J. M. Liu, Seong-Whan Lee, Offline Recognition of Chinese Handwriting by Multifeature and multilevel Classification, IEEE Transaction on Pattern Analysis and Machine Intelligence, 20:556-561, 1998.
[6] S.M. Chen, Similarity Measure between Vague Sets and between Elements, IEEE Trans on Systems, Man and Cybernetics, 27:153-158, 1997.

[7] S.M. Chen, Measures of Similarity between Vague Sets, Fuzzy Sets and Systems, 74:217-223, 1995.

[8] S. Madhvanath, G. Kim and V. Govindaraju, Chaincode Contour Processing for Handwritten Word Recognition, IEEE Transactions on Pattern Analysis and Machine Intelligence, 21:928-932, 1999.

[9] Z.Q. Lu, D.N. Jin, editor. Visual C++.NET Image Processing Programme, Tsinghua University Publishing Company, Beijing, 2006.

[10] S.H. Chen, L.X. Fu, editor. Applied Digital Image Processing, Science Press, Beijing, 2005.

[11] Mathematics Handbook, China Coal Industry Publishing House, Beijing, 1976. 